\title{
Differential effects of therapeutic regimens on specific classes of rheumatoid factor
}

\author{
RICHARD M POPE, JAMES LESSARD, AND EUGENE NUNNERY \\ From the Department of Medicine/Division of Clinical Immunology, University of Texas Health Science \\ Center, San Antonio, Texas, USA
}

SUMMARY This study was performed to define further the relationship of circulating IgM and IgG rheumatoid factor to the articular manifestations of rheumatoid arthritis. Patients with substantial clinical improvement $(>55 \%)$ or no improvement or worsening were chosen for study. Patients were further selected to include those treated with non-steroidal antiinflammatory drugs (NSAIDs) alone, or NSAIDs plus D-penicillamine or gold. Significant reductions of $\operatorname{IgM}(p<0.001)$ and IgG $(p<0.005)$ rheumatoid factor were seen only in those treated with gold who improved clinically. These observations suggest that in patients experiencing comparable degrees of clinical improvement the simultaneous alterations of circulating IgG and IgM rheumatoid factor observed are dependent upon the therapeutic regimen employed.

Key words: rheumatoid arthritis, articular manifestations.

Rheumatoid factors of the IgM, IgA, and IgG classes have been detected in the circulation and are produced locally by the synovium of patients with adult onset rheumatoid arthritis (RA) ${ }^{1-5}$ Although the presence of high concentrations of circulating rheumatoid factors has been associated with a poor prognosis, ${ }^{167}$ the relationship of circulating rheumatoid factors to disease activity, particularly the articular manifestations, remains poorly defined. In particular, the differing effects of various drug therapies on the relationship between serologic and articular manifestations have not been systematically examined. This study examines the relationship between changing articular manifestations and circulating IgM and IgG rheumatoid factors in RA patients treated with non-steroidal antiinflammatory drugs (NSAIDs) alone, or NSAIDs plus D-penicillamine or gold.

\section{Patients and methods}

\section{PAT I E N T S}

Patients (total 144) were examined prospectively in the outpatient clinics of the Medical Center Hospital and the Audie Murphy Veterans Administration

Accepted for publication 15 August 1985.

Correspondence to Dr Richard M Pope, Department of Medicine, Division of Rheumatology. Northwestern University School of Medicine, 303 E Chicago Avenue, Chicago, Illinois 60611, USA.
Hospital, San Antonio, TX. The eligibility requirements for this study were: $(a)$ a willingness to participate; $(b)$ definite or classical RA; ${ }^{8}(c)$ treatment with NSAIDs alone, or with NSAIDs plus gold or D-penicillamine (choice determined by patient's physician); $(d)$ patients remained on the drug of interest for at least three months; $(e)$ clear cut improvement or failure to improve on the described regimen (definition below).

In a previous study ${ }^{3}$ we observed substantial variability of clinical findings when patients improved only modestly (between 20 and $50 \%$ ). In order to avoid this variability and the attendant uncertainty patients were chosen for this study if their articular index improved more than 55\% (75\% for those on NSAIDs alone) or if it failed to improve ( $<10 \%$ improvement or worsening). More stringent criteria were applied to those treated with NSAIDs alone to improve our chances of observing a reduction of rheumatoid factor if this were due to clinical improvement on NSAIDs. Thus although the clinical criteria for inclusion were not strictly comparable, the selection process intentionally minimised the likelihood of observing a serologic trend associated with clinical improvement on NSAIDs alone. We reasoned that if a trend were not observed despite this bias it would be even more credible than if identically stringent criteria were applied to the group on NSAIDs. All patients were 
selected for study before performing the laboratory studies. In summary, the study groups included patients who improved or clearly did not improve on each of three therapeutic regimens.

The follow up evaluations used in this study for those who improved were not obtained at a uniform time after initiating therapy but at a time when comparable and substantial improvement (see criteria above) had occurred. For 'unimproved' subjects sampling was timed to correspond with that performed for the improved group. Thus although the follow up was not obtained at a uniform chronological time, it was obtained at a uniform time with regard to the clinical, therapeutic response. Nonetheless, as shown in Table 1, the mean time at follow up was similar for the three groups.

\section{CLINICAL EVALUATIONS PERFORMED}

An articular index was derived as previously described. ${ }^{3}$ Each swollen joint was multiplied by a weighted factor (f) which was dependent on the relative size of the joint. For the shoulder and knee, $f=3$; for the elbow and ankle, $f=2$; for all other joints, $f=1$. The articular index was derived by adding the number of swollen joints which had each been multiplied by the appropriate weighted factor. The articular index was chosen as the principal measure of clinical activity before initiating this study. For comparison, the joint count and sedimentation rate were included. The joint count was performed by summation of all the joints which were either swollen or tender to palpation or painful on standard range of motion. The erythrocyte sedimentation rate was performed by the Westergren method and reported in millimetres in the first hour.

RHEUMATOID FACTORS

Assays specific for IgM rheumatoid factor and $\mathrm{IgG}$ rheumatoid factor were performed as previously described. ${ }^{239}$ Briefly, normal IgG was employed at $50 \mu \mathrm{g} / \mathrm{ml}(\mathrm{mg} / \mathrm{l})$ to coat polystyrene wells overnight. For IgM rheumatoid factor, normal pooled human IgG was employed. For IgG rheumatoid factor, rabbit IgG was used. The importance of employing these specific antigens to ensure maximal sensitivity has been previously reported. ${ }^{10}$ Any remaining adherent sites were blocked with $1 \%$ bovine serum albumin. Diluted patient sera were then added to the wells and incubated overnight. Before performing the IgG rheumatoid factor radioimmunoassay the sera were first pepsin digested in order to abrogate the effects of $\operatorname{IgM}$ and $\operatorname{IgA}$ rheumatoid factor. ${ }^{9}$ Individual classes of rheumatoid factor were then quantified with class specific antisera: $F\left(a^{1}\right)_{2}$ antihuman IgM ( $\mu$ specific) for IgM rheumatoid factor and $F\left(a b^{1}\right)_{2}$ rabbit antihuman $F a b(\gamma$ specific) for IgG rheumatoid factor. The upper limits of normal were determined by employing 20 simultaneously examined normal sera and purified polyclonal IgG rheumatoid factor and IgM rheumatoid factor. The upper limit of normal for IgM rheumatoid factor was $2.9 \mu \mathrm{g} / \mathrm{ml}$ and for IgG rheumatoid factor, $29 \mu \mathrm{g} / \mathrm{ml}$.

CALCULATIONS AND STATISTICS

Percentage change in study variables was determined by the formula:

$$
\text { Percentage change }=\frac{\text { final value }- \text { initial value }}{\text { higher value }}
$$

A negative percentage change was indicative of improvement and a positive value of worsening. This formula allowed all changes to be compared with the maximal value for each variable.

The significance of differences between group means was determined by Student's $t$ test. A paired $t$ test was used for matched pairs. Correlation coefficients were determined with Spearman's rank correlation test.

\section{Results}

PATIENT DESCRIPTION

Clinical characteristics of the patient groups are presented in Table 1. Therapeutic groups were comparable in number, ranging from 13 to 16

Table 1 Patient description

\begin{tabular}{|c|c|c|c|c|c|c|}
\hline Group therapy & No & $\begin{array}{l}\text { Therapeutic effectiveness } \\
\text { subgroup (No) }\end{array}$ & $\begin{array}{l}\text { Mean age } \\
\text { (range) (years) }\end{array}$ & $\begin{array}{l}\text { Mean duration (years) } \\
\text { of } R A \text { (range) }\end{array}$ & Gender & $\begin{array}{l}\text { Mean duration (months) } \\
\text { of follow up (range) }\end{array}$ \\
\hline NSAIDs only & 16 & $\begin{array}{l}\text { Improved (8) } \\
\text { Not improved (8) }\end{array}$ & $\begin{array}{l}57(37-65) \\
57(42-71)\end{array}$ & $\begin{array}{r}7(0 \cdot 3-15) \\
12(0 \cdot 3-20)\end{array}$ & $\begin{array}{l}6 \mathrm{~F}, 2 \mathrm{M} \\
5 \mathrm{~F}, 3 \mathrm{M}\end{array}$ & $\begin{array}{l}8 \cdot 3(5-13) \\
8 \cdot 4(3-17)\end{array}$ \\
\hline D-Penicillamine & 13 & $\begin{array}{l}\text { Improved (8) } \\
\text { Not improved (5) }\end{array}$ & $\begin{array}{l}56(41-64) \\
53(50-59)\end{array}$ & $\begin{array}{r}15(8-30) \\
8(5-10)\end{array}$ & $\begin{array}{l}4 \mathrm{~F}, 4 \mathrm{M} \\
3 \mathrm{~F}, 2 \mathrm{M}\end{array}$ & $\begin{array}{l}11 \cdot 3(6-17) \\
11 \cdot 4(6-17)\end{array}$ \\
\hline Gold & 15 & $\begin{array}{l}\text { Improved (9) } \\
\text { Not improved (6) }\end{array}$ & $\begin{array}{l}54(36-65) \\
54(40-71)\end{array}$ & $\begin{array}{l}6(0 \cdot 3-20) \\
9(2-23)\end{array}$ & $\begin{array}{l}6 \mathrm{~F}, 3 \mathrm{M} \\
4 \mathrm{~F}, 2 \mathrm{M}\end{array}$ & $\begin{array}{l}9 \cdot 3(5-14) \\
9 \cdot 3(4-15)\end{array}$ \\
\hline
\end{tabular}


patients each. The therapeutic effectiveness subgroups ranged from five to nine patients each. The mean ages of the patients ranged from 53 to 57 years. The mean duration of rheumatoid arthritis before entry in this study was broad, ranging from four months to 30 years. The mean duration of follow up was comparable between the subgroups.

CLINICAL AND LABORATORY CHANGE OF THE S U B G ROU P S

Clinical and laboratory data at the initial visit for each subgroup are recorded in Table 2 . The initial clinical and laboratory measures of disease activity were generally higher in the patients who improved compared with those who failed to improve. The only baseline differences which were statistically significant, however, were the articular index of the improved and unimproved patients receiving penicillamine $(\mathrm{p}<0.02)$ and the ESR of the improved $v$ unimproved subjects receiving gold $(p<0.05)$.

The mean changes between the initial and final observations for the clinical and serologic variables are presented in Figs 1-3. For the NSAID subgroup that improved (Fig. 1) amelioration of the clinical parameters ranged from $86 \%(p<0.001)$ for the articular index and $61 \%(\mathrm{p}<0.002)$ for the joint count to $32 \%(p=N S)$ for the erythrocyte sedimentation rate. Reductions of rheumatoid factor concentrations in this subgroup were modest: only $19 \%$ for IgM RF and $25 \%$ for IgG RF. In the

Table 2 Initial clinical and laboratory values for the different therapeutic groups and subgroups

\begin{tabular}{llllll}
\hline & Articular index & Joint count & ESR $(\mathrm{mm} / \mathrm{lst} \mathrm{h})$ & IgM RF $(\mu \mathrm{g} / \mathrm{ml})$ & IgG RF $(\mu \mathrm{g} / \mathrm{ml})$ \\
\hline NSAIDs & & & & & \\
$\quad$ Improved & $29 \pm 14^{*}(16-50)$ & $25 \pm 10(18-42)$ & $60 \pm 43(17-139)$ & $50 \pm 38(11-135)$ & $1388 \pm 1408(112-3110)$ \\
$\quad \begin{array}{l}\text { Unimproved } \\
\text { D-Penicillamine }\end{array}$ & $18 \pm 11(7-35)$ & $22 \pm 12(10-41)$ & $48 \pm 29(4-97)$ & $37 \pm 38(0-109)$ & $346 \pm 528(13-1584)$ \\
$\quad$ & & & & \\
$\quad$ Improved & $34 \pm 12(18-52)$ & $27 \pm 11(18-52)$ & $63 \pm 29(27-98)$ & $37 \pm 15(20-62)$ & $818 \pm 832(218-2708)$ \\
$\quad$ Unimproved & $14 \pm 10(5-31)$ & $13 \pm 11(5-32)$ & $42 \pm 30(10-75)$ & $36 \pm 11(22-46)$ & $615 \pm 567(154-1524)$ \\
Gold & & & & & \\
$\quad$ Improved & $32 \pm 12(17-59)$ & $28 \pm 11(17-46)$ & $66 \pm 26(27-103)$ & $43 \pm 8(33-55)$ & $596 \pm 599(112-1970)$ \\
$\quad$ Unimproved & $19 \pm 12(0-33)$ & $18 \pm 10(4-28)$ & $37 \pm 17(14-52)$ & $37 \pm 19(8-51)$ & $490 \pm 356(127-1156)$ \\
\hline
\end{tabular}

*Values are mean $\pm \mathrm{SD}$; the values in the parentheses represent the ranges.
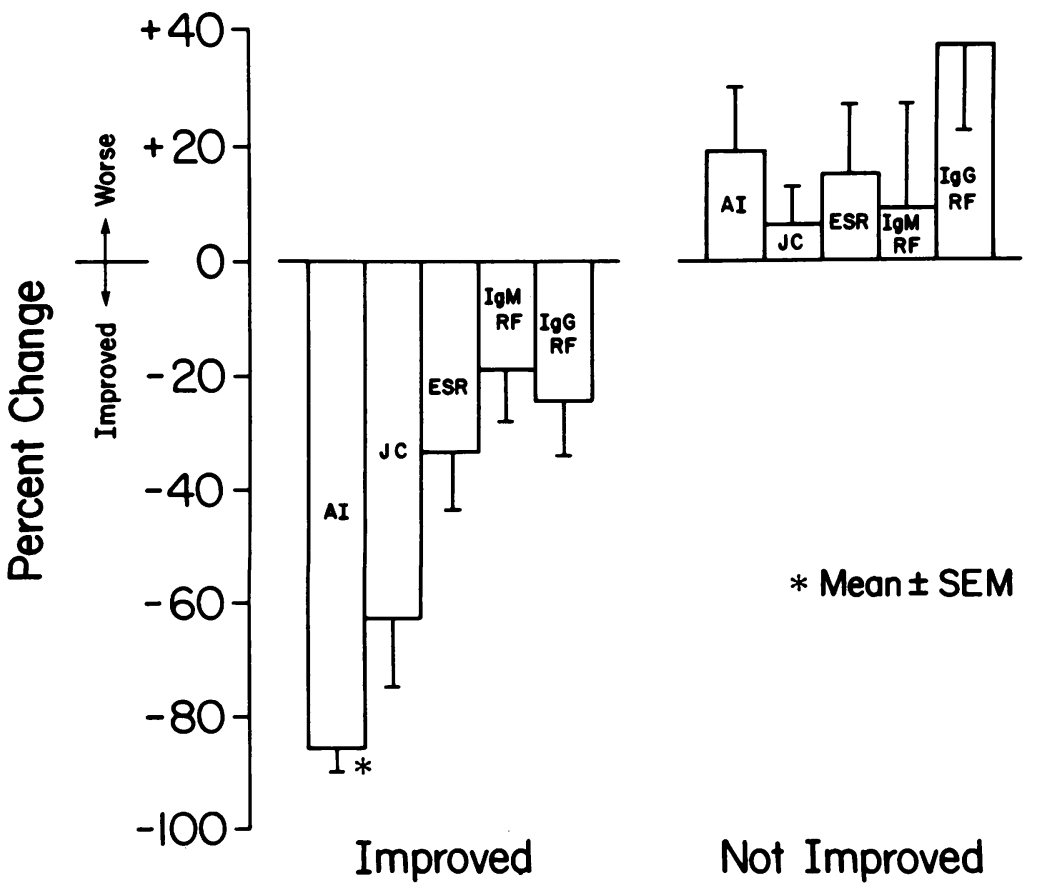

Fig. 1 Change of clinical and laboratory parameters in patients treated with NSAIDs alone. The values for the patients who improved are presented in the left of the figure and for those who did not improve on the right. The values are presented as the mean \pm 1 SEM. The abbreviations employed are: $A I=$ articular index; $J C=$ joint $*$ Mean \pm SEM count; $E S R=$ erythrocyte sedimentation rate; IgM $R F=I g M$ rheumatoid factor; IgG $R F=I g G$ rheumatoid factor. 


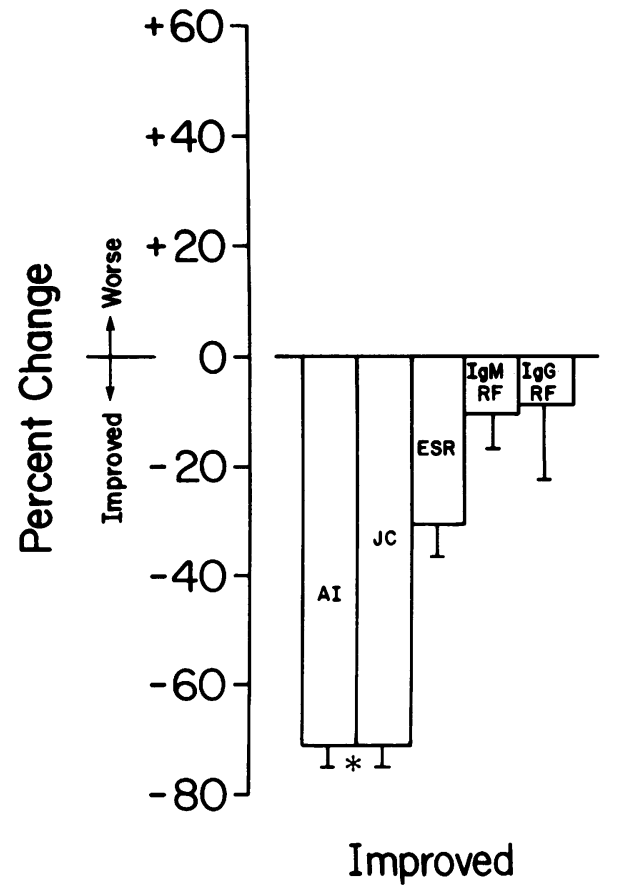

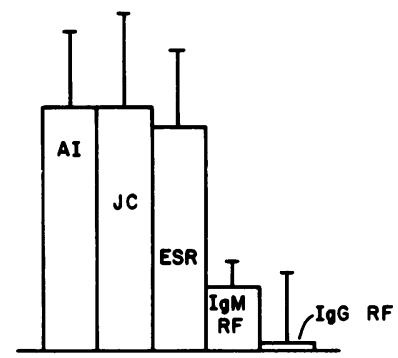

Fig. 2 Change of clinical and laboratory parameters in patients treated with NSAIDs plus D-penicillamine. See Fig. 1 for details.

Fig. 3 Change of clinical and laboratory parameters in patients treated with NSAIDs plus gold. See Fig. 1 for details.

\section{Not Improved}

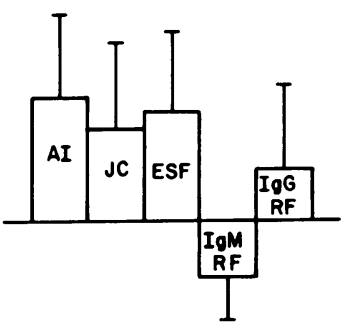

* Mean \pm SEM

Not Improved 
unimproved subgroup deterioration of clinical activity ranged from $19 \%$ for the articular index to $6 \%$ for the joint count. In this subgroup IgM RF increased $9 \%$, while the IgG RF increased a mean of $37 \%$. Neither class of rheumatoid factor changed significantly in either subgroup.

In the groups treated with NSAIDs plus Dpenicillamine (Fig. 2) improvement was somewhat less and deterioration generally somewhat worse than noted for those on NSAIDs alone. In the subgroup that improved the articular index and joint count both decreased $71 \%(p<0.001)$, while the ESR decreased $31 \%(p<0.005)$. In the subgroup that failed to improve the clinical manifestations deteriorated by $40-44 \%$. In neither the improved nor unimproved subgroups did the IgM RF or the IgG RF change significantly, with mean changes of $11 \%$ or less.

The changes noted in the patients treated with gold (Fig. 3) were somewhat different. Clinical improvement ranged from a mean of $83 \%$ $(p<0.001)$ for the articular index and $74 \%$ $(p<0.001)$ for the joint count to $57 \%(p<0.005)$ for the erythrocyte sedimentation rate. In this subgroup the mean reduction of IgM RF and IgG RF were comparable to the clinical changes, $59 \%(p<0.001)$ and $70 \%(p<0.005)$ respectively. In those patients who failed to improve on gold all parameters deteriorated modestly, with the exception of IgM rheumatoid factor which decreased slightly.

Table 3 Relationship between change of clinical and laboratory variables

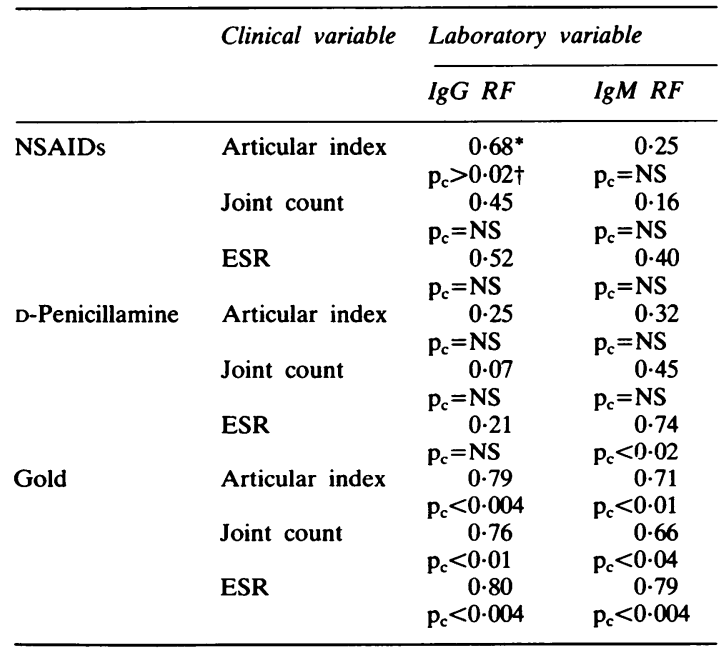

*Correlation coefficient determined by Spearman's rank correlation test.

† Significance of the correlation coefficient corrected $\left(p_{c}\right)$ for the number of laboratory variables examined.
RELATIONSHIP BETWEEN CHANGE OF CLINICAL VARIABLES AND CHANGE OF RF To examine the relationship between the change of the clinical variables and the change of IgM and IgG rheumatoid factor data both for those patients who improved and those who failed to improve were combined within each of the three therapeutic groups (Table 3). These relationships must be evaluated with caution since the data were obtained from the two extremes of therapeutic responsiveness. Nonetheless, change of RF correlated poorly with change of clinical activity in those treated with NSAID alone or with D-penicillamine. In contrast, IgM RF, and to a greater extent IgG RF, correlated significantly with change of clinical activity in those treated with gold.

\section{Discussion}

This study examined the relationship between the articular manifestations of rheumatoid arthritis and concentrations of circulating IgM and IgG RFs. Only in those patients treated with gold was a significant reduction of both classes of rheumatoid factors observed in patients preselected on the basis of clinical improvement. Although rheumatoid factors decreased in the patients treated with NSAIDs alone or with NSAIDs plus D-penicillamine, these changes were not significant even in the face of highly significant clinical improvement which was comparable to the improvement of those treated with gold.

In previous studies rheumatoid factor reduction has been associated with certain forms of therapy. One study ${ }^{11}$ observed an increase of IgM rheumatoid factor after cessation of NSAIDs for two weeks. After reinstitution of NSAIDs for six weeks a significant reduction of circulating IgM RF was noted. No objective measurement of disease activity was made in this study. In our study, even in the group of patients preselected on the basis of dramatic clinical improvement $(81 \%)$, the reduction of both IgG and IgM RFs failed to achieve statistical significance. However, our patients were taking a variety of NSAIDs and this therapy was not routinely altered at the time of entry. The previous study ${ }^{11}$ employed a single agent and therapy was modified at the onset of the study. Differences of design probably account for the discrepancies between these two studies.

Patients treated with D-penicillamine have often, ${ }^{12-14}$ but not uniformly, ${ }^{15}{ }^{16}$ experienced significant reductions of circulating rheumatoid factor during therapy. Although most studies employing agglutination techniques have noted a reduction of rheumatoid factor with D-penicillamine therapy, reduction has not always been significant even in the 
face of significant clinical improvement ${ }^{15} 16$ and has often been associated with doses of D-penicillamine which are highly toxic. The study by Wernick et al. ${ }^{12}$ employing radioimmunoassays noted a significant reduction of circulating IgM RF in those treated with D-penicillamine. This study ${ }^{12}$ included all patients regardless of clinical response. In our patients who improved no significant reduction of either IgG or IgM RF was observed, despite a mean $71 \%$ clinical improvement. Wernick et al. ${ }^{12}$ noted significant reduction of IgM RF by nine months regardless of the dose employed ( 250 or $750 \mathrm{mg} /$ day). Our patients were followed up for a mean of 11.3 months. Wernick et al. ${ }^{12}$ did note, however, that the reduction of IgM RF was greater and occurred sooner in those on the higher dose $(750 \mathrm{mg} /$ day $)$. The majority of our patients received a maximally tolerated dose (mean $412 \pm 71 \mathrm{mg} / \mathrm{day}$ ), and none received less than $250 \mathrm{mg} /$ day.

The most dramatic modulation of serologic variables noted in this study was in those who improved clinically during treatment with gold. Despite the fact that the clinical amelioration was comparable with that of the other two therapeutic subgroups, only in those treated with gold were significant reductions of IgM and IgG RF noted. Previous studies have not examined the effects of class specific radioimmunoassays employing gold. Nonetheless, even with less specific agglutination techniques, many ${ }^{17-19}$ but not all ${ }^{20}$ studies have observed decreased rheumatoid factor in patients treated with gold. ${ }^{18-20}$ In one study ${ }^{18} 33 \%$ of patients actually became seronegative. Another study $^{19}$ observed reduced rheumatoid factor titres, despite the therapeutic result, though the titres decreased further in the patients who experienced excellent clinical responses. In contrast with those experiencing clinical improvement, we did not observe a significant reduction of either IgM or IgG $\mathrm{RF}$ in those who failed to improve. Therefore, gold did not cause a reduction of rheumatoid factor in all patients since significant reductions occurred only in the subgroup that experienced clinical improvement. The fact that the IgM and IgG RFs of the NSAID group alone and the NSAIDs plus Dpenicillamine therapeutic improvement subgroup did not fall significantly in the face of clinical improvement comparable with the improvement of those treated with gold suggests that changes of rheumatoid factor were not simply non-specific secondary to clinical improvement. If rheumatoid factors were generated in response to synovial inflammation they should have been reduced comparably in all the clinical improvement subgroups since they all experienced similar degrees of disease remission.

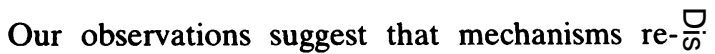
sponsible for clinical improvement may have been $\Rightarrow$ different in the three therapeutic groups. In those $\stackrel{\vec{f}}{9}$ treated with gold perhaps a true immunosuppressive 0 effect, at least for the production of rheumatoid factor, occurred. In the other two therapeutic $\frac{\bar{s}}{5}$ subgroups that improved no significant reductions of $\underset{\complement}{\mathbb{D}}$ rheumatoid factors occurred, suggesting that different mechanisms may have been responsible.

$\mathrm{We}^{3}$ and others ${ }^{21}$ have observed that circulating. IgG RF concentration more accurately reflects $\vec{\omega}$ articular activity than other classes of rheumatoid factor or immune complexes. However, the associa- $\frac{0}{2}$ tions noted in these studies were not strong enough to be clinically useful. Although, as mentioned, the correlations observed in this study must be viewed ${ }_{\vec{t}}^{\omega}$ with reservation, the clinical utility of routinely ${ }_{\omega}^{\infty}$ measuring IgM and IgG RFs would appear to beo limited. Only in those treated with gold were the associations of the articular and laboratory changes 3 reliable. This lack of association has previously been $\frac{0}{3}$ noted in those treated with D-penicillamine and $\stackrel{\Im}{5}$ those treated with total nodal irradiation. ${ }^{22} 23$ Our $\overrightarrow{0}$ study did not consider the usefulness of $\operatorname{IgG}$ or $\operatorname{IgM}_{\sigma}^{\infty}$ RF for following extra-articular disease such as vasculitis, where they may be of more value. ${ }^{24} 25$ Ito will be of interest to observe the long term prognostic significance of the reduction of IgM or IgG RF noted in some of our patients.

We thank Ethel Kern for her excellent assistance in the preparation of this manuscript. We thank Dr Richard Deyo for his thoughtful 3 review and helpful comments. This work was supported in part by a? Clinic Research Center grant from the Arthritis Foundation.

\section{References}

1 Kellgren J H, O'Brien W M. On the natural history of $\frac{0}{}$ rheumatoid arthritis in relation to the sheep cell agglutination. test (SCAT). Arthritis Rheum 1962; 5: 115.

2 Cecere F, Lessard J, McDuffy S, Pope R M. Evidence for the local production and utilization of immune reactants in rheumatoid arthritis. Arthritis Rheum 1982; 25: 1307-15.

3 Lessard J, Nunnery E, Cecere F, McDuffy S, Pope RM.D Relationship between the articular manifestations of rheu-O음 matoid arthritis and circulating immune complexes detected by three methods and specific classes of rheumatoid factors. J Rheumatol 1983; 10: 411-7.

4 Egeland T, Lea T, Saari G, Mellbye O J, Natvig J B. N Quantitation of cells secreting rheumatoid factor of $\operatorname{IgG}, \operatorname{IgA}, \mathrm{N}$ and IgM class after elution from rheumatoid synovial tissue. $\omega$ Arthritis Rheum 1982; 25: 1445-50.

5 Koopman W J, Miller R K, Crago S S, Mestecky Jô Schrohenloher R E. IgA rheumatoid factor: evidence for independent expression at local sites of tissue inflammation. Ann N Y Acad Sci 1983; 409: 258-71.

6 Jacoby R K, Jayson M I V, Cosh J A. Onset, early stages and prognosis of rheumatoid arthritis: a clinical study of 100 patients $\frac{\text { (D) }}{8}$ with 11-year follow-up. Br Med J 1973; ii: 96-100.

7 Masi A T, Maldonado-Cocco J A, Kaplan S B, et al. $\frac{\text { (D) }}{Q}$ Prospective study of the early course of rheumatoid arthritis in young adults: comparison of patients with and without rheuma 
toid factor positivity at entry and identification of variables correlating with outcome. Semin Arthritis Rheum 1976; 5: 299-326.

8 Ropes M W, Bennett G A, Cobb S, Jacox R, Jessar R A. 1958 revision of diagnostic criteria for rheumatoid arthritis. Bull Rheum Dis 1958; 9: 175-6.

9 Pope R M, McDuffy S J. IgG rheumatoid factor: relationship to seropositive rheumatoid arthritis and absence in seronegative disorders. Arthritis Rheum 1979; 22: 988-98.

10 Pope R M, McDuffy S J. IgG rheumatoid factor: analysis of various aspects of $\mathrm{IgG}$ for detection by radioimmunoassay. $J$ Lab Clin Med 1981; 97: 842-53.

11 Goodwin J S, Ceuppens J L, Rodriguez M A. Administration of non-steroidal anti-inflammatory agents in patients with rheumatoid arthritis. JAMA 1983; 250: 2485-8.

12 Wernick R, Merryman P, Jaffe I, Ziff M. IgG and IgM rheumatoid factors in rheumatoid arthritis: quantitative response to penicillamine therapy and relationship to disease activity. Arthritis Rheum 1983; 26: 593-8.

13 Jaffe I A. The effect of penicillamine on the laboratory parameters in rheumatoid arthritis. Arthritis Rheum 1965; 8: $1064-79$.

14 Bluestone R, Goldberg L S. Effect of D-penicillamine on serum immunoglobulins and rheumatoid factor. Ann Rheum Dis 1973; 32: 50-2.

15 Andrews F M, Golding D N, Freeman A M, et al. Controlled trial of D-penicillamine in severe rheumatoid arthritis. Lancet 1973; i: $275-80$.

16 Dixon J S, Pickup M E, Lowe J R, Hallett C, Lee M R, Wright $V$. Discriminatory indices of response of patients with rheuma- toid arthritis treated with D-penicillamine. Ann Rheum Dis 1980; 39: 301-11.

17 Sharp J T, Lidsky M D, Duffy J, et al. Comparison of two dosage schedules of gold salts in the treatment of rheumatoid arthritis. Arthritis Rheum 1977; 20: 1179-87.

18 Lorber A, Simon T, Leeb J, Peter A, Wilcox S. Chrysotherapy. Arthritis Rheum 1978; 21: 785.

19 Sharp J T, Lidsky M D, Duffy J. Clinical responses during gold therapy for rheumatoid arthritis. Arthritis Rheum 1982; 25: 540-9.

20 Srinivasan R, Miller B L, Paulus H E. Long-term chrysotherapy in rheumatoid arthritis. Arthritis Rheum 1979; 22: 105-10.

21 McDougal J S, Hubbard M, McDuffie F C, et al. Comparison of five assays for immune complexes in the rheumatic diseases. Arthritis Rheum 1982; 25: 1156-66.

22 Kotzin B L, Strober S, Engleman E G, et al. Treatment of intractable rheumatoid arthritis with total lymphoid irradiation. $N$ Engl J Med 1981; 305: 969-76.

23 Trentham D E, Belli J A, Anderson R J, et al. Clinical and immunologic effects of fractionated total lymphoid irradiation in refractory rheumatoid arthritis. $N$ Engl $J$ Med 1981; 305: 976-82.

24 Scott D G, Bacon P A, Allen C, Elson C J, Wallington T. IgG rheumatoid factor, complement and immune complexes in rheumatoid synovitis and vasculitis: comparative and serial studies during cytotoxic therapy. Clin Exp Immunol 1981; 43: 54-63.

25 Quismorio F P, Beardmore T, Kaufman R L, Mongan E S. IgG rheumatoid factors and anti-nuclear antibodies in rheumatoid vasculitis. Clin Exp Immunol 1983; 52: 333-40. 